\title{
Evolutionary Selection of Features for Neural Sleep/Wake Discrimination
}

\author{
Peter Dürr, Walter Karlen, Jérémie Guignard, Claudio Mattiussi, and Dario Floreano
}

Laboratory of Intelligent Systems, Ecole Polytechnique Fédérale de Lausanne, 1015 Lausanne, Switzerland

Correspondence should be addressed to Peter Dürr, peter.duerr@epfl.ch

Received 15 November 2008; Accepted 19 February 2009

Recommended by Janet Clegg

\begin{abstract}
In biomedical signal analysis, artificial neural networks are often used for pattern classification because of their capability for nonlinear class separation and the possibility to efficiently implement them on a microcontroller. Typically, the network topology is designed by hand, and a gradient-based search algorithm is used to find a set of suitable parameters for the given classification task. In many cases, however, the choice of the network architecture is a critical and difficult task. For example, hand-designed networks often require more computational resources than necessary because they rely on input features that provide no information or are redundant. In the case of mobile applications, where computational resources and energy are limited, this is especially detrimental. Neuroevolutionary methods which allow for the automatic synthesis of network topology and parameters offer a solution to these problems. In this paper, we use analog genetic encoding (AGE) for the evolutionary synthesis of a neural classifier for a mobile sleep/wake discrimination system. The comparison with a hand-designed classifier trained with back propagation shows that the evolved neural classifiers display similar performance to the hand-designed networks, but using a greatly reduced set of inputs, thus reducing computation time and improving the energy efficiency of the mobile system.
\end{abstract}

Copyright ( $\odot 2009$ Peter Dürr et al. This is an open access article distributed under the Creative Commons Attribution License, which permits unrestricted use, distribution, and reproduction in any medium, provided the original work is properly cited.

\section{Introduction}

The traditional way to craft an artificial neural network (ANN) for a classification task is to hand design a network topology and to find a set of network parameters using a gradient-based error-minimization algorithm such as back propagation [1]. However, in real-world applications, such as the classification of biomedical signals, the network topology can be difficult to design by hand. Additionally, in many cases, it is desirable to minimize the computational cost of the network, for example, by reducing the number of inputs used by the classifier. Evolutionary methods for the design of ANNs can provide an answer to both issues [2]. In this paper, we study the application of a neuroevolutionary method called analog genetic encoding (AGE) [3] to the problem of synthesis and optimization of neural networks for the processing of biological signals aimed at sleep and wake classification.

Continuous monitoring of the sleep/wake state of highrisk professionals such as pilots, truck drivers, or shift workers can potentially decrease the risk of accidents and help scheduling breaks and resting times. However, implementing such a classification in a wearable device is a challenging task. Limited energy and processing resources as well as the increased noise level due to movement artifacts and a constantly changing environment put tight restrictions on the choice of sensors and algorithms. Traditionally, the states of sleep and wake are classified based on the analysis of brain wave patterns (EEG) [4]. EEG recording requires gluing electrodes to the scalp and is typically susceptible to different sources of noise. Methods relying on EEG measurements are thus more suited for sleep analysis in controlled hospital environments than for mobile applications.

For mobile sleep/wake pattern screening, a commonly used technique is actigraphy [5]. In actigraphy, the acceleration of the wrist of the subject is recorded, and phases of weak activity-as judged by the levels of accelerationare classified as sleep. Actigraphy devices can be small, inexpensive, and low power, which makes them suitable for mobile applications. However, as the signals provided by actigraphy devices are not directly linked to physiological states, it is difficult to derive a reliable prediction from 
them. Activities characterized by low levels of motion, such as reading or watching $\mathrm{TV}$, are often misclassified as sleep [6]. In $[7,8]$, we have suggested to use electrocardiogram (ECG) and respiratory effort (RSP) signals for wearable sleep/wake classification (see Figure 1). Both signals depend on properties of the activity of the autonomous nervous system, which differ in sleep and wake [9]. Furthermore, they are measurable with portable sensor systems such as the Heally system (see Figure 2, Koralewski Industrie Elektronik, Celle, Germany).

An additional difficulty is that the generation of a set of labeled data for the training of the classifier is typically a time-consuming activity for both the subjects from whom data is collected and the technicians who must label the data [7]. It is thus desirable to design a classifier that can be trained on a set of data and can then be used on further subjects without additional training. In [7], we have shown that using the frequency content of the ECG and RSP signals as input features for a single layer ANN, a mean accuracy of $86.7 \%$ can be achieved when the network was trained and tested on data obtained from different subjects. A limitation of the hand-designed ANN used in [7] is its large number of inputs. Some of these inputs are presumably redundant and might not contribute significantly to the classifier performance. For the targeted mobile application, the power consumption of the classifier is critical. In order to reduce processing time and thus power consumption for mobile applications, it would be desirable to minimize the number of inputs. In this paper, we show how to automatically synthesize networks that use a small subset of the spectral components associated with the signals as inputs while maintaining the performance of the classifier.

\section{Evolutionary Synthesis of Neural Networks}

Neural networks can be described as directed graphs, where the nodes represent a neuron model, and the edges of the graph are associated with the weighted connections between the neurons, the so-called synaptic weights. The design of a network for a particular task thus involves the choice of the topology of the graph (i.e., the network architecture) and a suitable set of numerical parameters (i.e., the synaptic weights and the parameters of the neuron model). The automatic synthesis of the topology and parameters of a neural network requires a computer representation for both aspects of the network, combined with an algorithm capable of performing a search in the space defined by this representation. Evolutionary algorithms have been extensively used to evolve neural classifiers because these algorithms can combine a flexible representation with a high potential of stochastic exploration of the search space [10-13].

The simplest approach to this, the so-called direct encoding, represents all the neurons, synaptic connections, and parameters of the network explicitly (see, e.g. [14-16]). This has the advantage that the resulting networks can easily be decoded from the genome. However, with increasing size of the network, the length of the corresponding genome grows rapidly, which can affect the evolvability. In order to mitigate this problem, it has been suggested to encode a program or a sequence of instructions that, when executed, builds the network. This developmental encoding can lead to very compact representations of large networks (see, e.g., $[17,18])$. However, the definition of a set of mutation and recombination operators which guarantees that only valid networks are generated during the search is typically very difficult.

A promising alternative to direct and developmental representations that is getting more and more popular is implicit encoding [19-23]. In this paper, we use an implicit representation called analog genetic encoding (AGE). AGE has been shown to be very effective for the automatic synthesis of various kinds of networks and, in particular, of neural networks [2, 3, 24-26].

The concept of implicit encodings like AGE is loosely inspired by the working of biological gene regulatory networks (GRNs). In biological GRNs, the interactions between the genes are not explicitly encoded in the genome but follow implicitly from the physical and chemical environment in which the genome is immersed. Simplifying a bit the picture, the activation of a biological gene depends on the interaction of molecules produced by another gene with parts of the activated gene called regulatory regions (Figure 3(a)). AGE abstracts this picture and defines an artificial genome composed of sequences of characters, for example, the uppercase ASCII set (Figure 3(b)). Similar to the function of promoter and terminal regions in GRNs, special sequences (the so-called tokens) identify regions of the artificial genome as artificial genes, which encode individual neurons. The sequences delimited by the tokens are interpreted analogous to coding regions and regulatory regions in biological GRNs. The strength of the connection between two neurons is implicitly determined by the coding region of one neuron and the regulatory region of another neuron via a function called interaction map. The interaction map can be seen as an abstraction of the biochemical process of gene regulation. It takes sequences of characters as arguments and outputs a real-valued connection strength. In our implementation, this is obtained by mapping the local alignment score [27] of the two sequences exponentially to the interval that spans all possible weight values (see [24]).

In summary, the AGE genome can be decoded first by extracting the neurons with the associated (coding and regulatory) sequences of characters. This is realized by scanning the genome for tokens which indicate the presence of a neuron $(\mathrm{GN})$. Together with predefined terminator sequences (TE), these tokens delimit the part of the genome associated with the respective neuron. The enclosed sequences of characters are interpreted as the coding and regulatory sequences of the respective neuron. Subsequently, the interaction map $I$ can be applied to all pairs of coding and regulatory sequences to obtain the synaptic weights $w_{i j}$ connecting the neurons (see Figure 4).

In this framework, there are several different possibilities to implement connections from external inputs to external outputs (see [28] for more details). Here, we encoded the coding sequences associated to the input neurons and the regulatory sequences associated to the output neurons in 


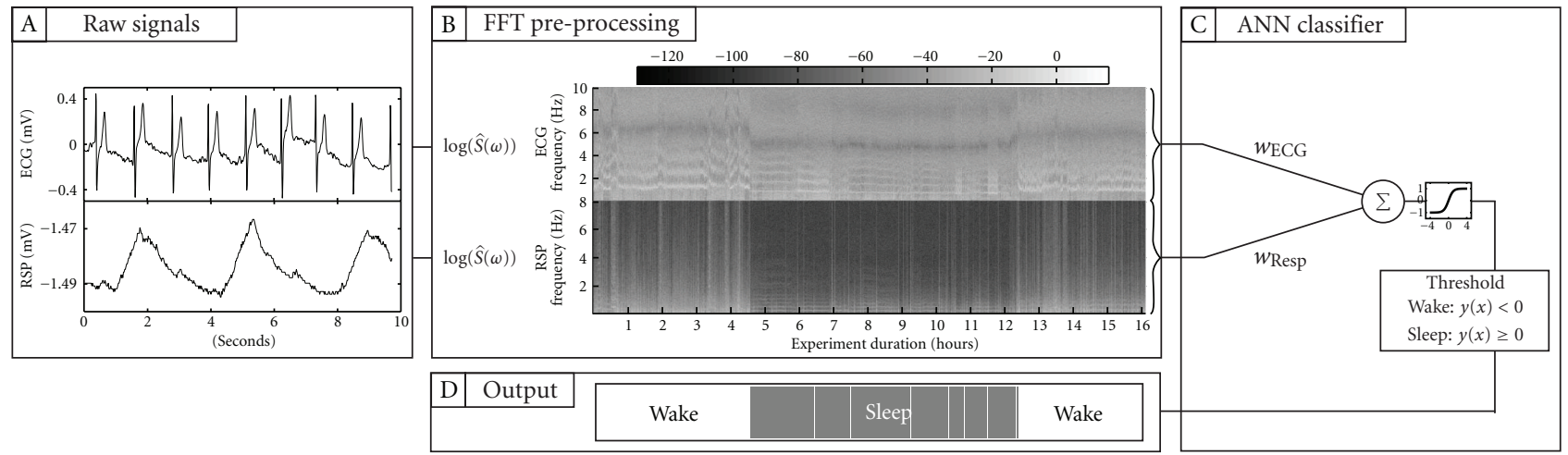

FIGURE 1: Overview of the sleep/wake classification system. (a) The raw electrocardiogram (ECG) and respiratory effort (RSP) signals are cut into windows of 40.06 seconds. (b) A short-time fast Fourier transformation (FFT) is used to calculate the spectral power of the windowed signals. (c) The resulting frequency data are fed to a feed-forward artificial neural network (ANN) and (d) a symmetric threshold classifies the ANN output into sleep or wake state estimates.

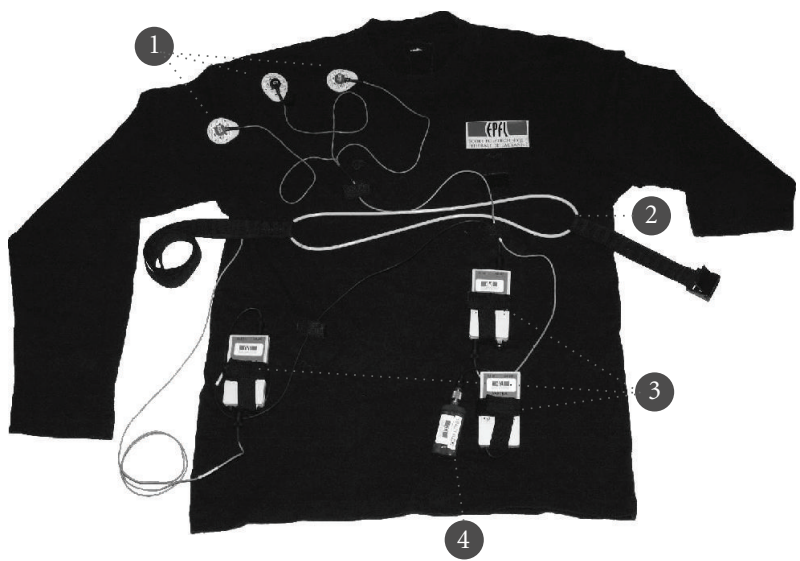

Figure 2: Portable Heally recording system mounted on a shirt. (1) ECG gel electrodes; (2) inductive belt sensor; (3) electronics modules; (4) NiMH battery.

separated parts of the genome (see Figure 5). In this case, the connections from the input neurons to the network can be obtained by applying the interaction map to all pairs of coding sequences (associated with the input neurons and the hidden neurons) and regulatory regions (associated to the hidden neurons and the output neuron). Note that the interaction map can associate a null weight value, thus leaving the respective neurons unconnected. When this feature is applied to the connections stemming from the input neurons, it gives evolution the freedom to select a subset of the set of inputs that contains the information necessary to realize the classification task.

As the sequences which define the strength of the synaptic connections can have a variable length and the interaction map is defined to operate on sequences of arbitrary length, a large class of genetic operators can be used to alter the network. In particular, we use the biologically plausible insertion, substitution, and deletion of characters and the transposition, duplication, and deletion of fragments of genome. The changes in the genome caused by these mutation operators can reflect both changes in the parameters of the network as well as changes in the network structure. For example, the insertion of a character in the genome can lead to a change of the synaptic weight connecting a particular input to the output neuron. The deletion of a fragment of genome associated with an input of the network can lead to the removal of this particular input from the network. Furthermore, the number of hidden neurons in the network can increase (e.g., after a genome fragment duplication) or decrease (e.g., after a character substitution) over the course of evolution. Given the fact that parts of the genome can be noncoding (i.e., they are not part of the description of a neuron) and that the interaction map is defined to be highly redundant, many mutations do not have an effect on the decoded networks. This allows for a high neutrality in the search space, which can improve evolvability [29].

\section{Experiments}

To compare the performance of the classical approach to classifier synthesis and training with the state-of-the-art neuroevolution method based on AGE, we performed a set of experiments, where we compared the performance of a neural network with fixed hand-designed topology and variable weights trained with back propagation, with that of neural networks synthesized with an evolutionary algorithmbased on AGE. As anticipated, we are interested in the performance in a sleep/wake detection task, where data from a set of users is available for network synthesis and training, but the performance is expected to generalize to additional users. We thus investigated the performance of the two methods when trained on ECG and RSP data collected on multiple subjects, and tested on data from a different subject.

3.1. Data. The data used in the following experiments are identical with those described in [8], where a hand-designed classifier with back propagation was used. They stem from 


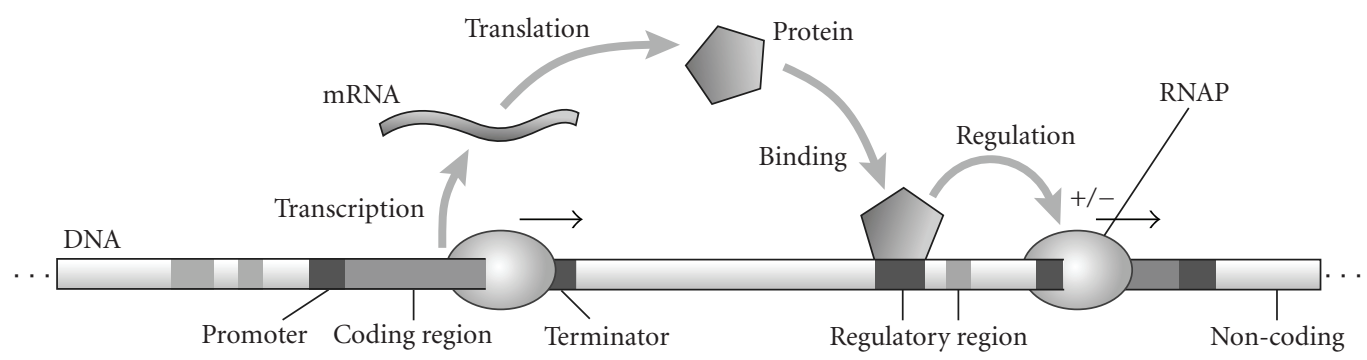

(a) Transcriptional regulation

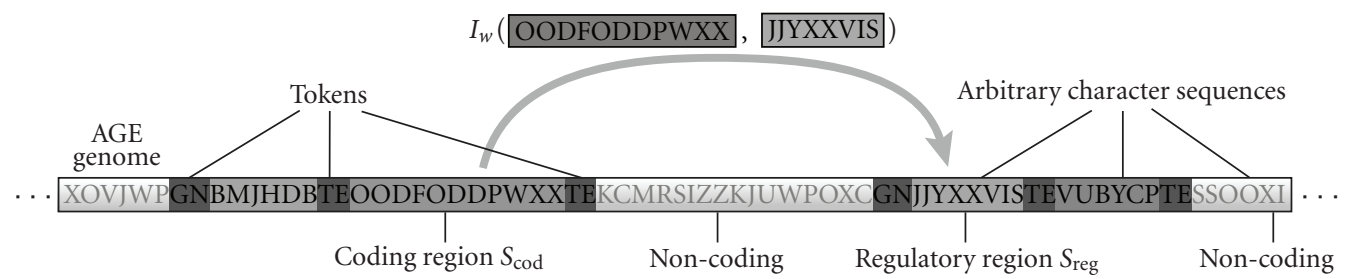

(b) The AGE abstraction

FIGURE 3: (a) In biological gene networks, the link between genes is realized by molecules that are synthesized from the coding region of one gene and interact with the regulatory region of another gene. (b) Analog genetic encoding abstracts this mechanism using an artificial genome containing markers that identify the artificial genes, and an interaction map that creates links between pairs of artificial genes by associating with them a numerical value that represents the strength of the link.

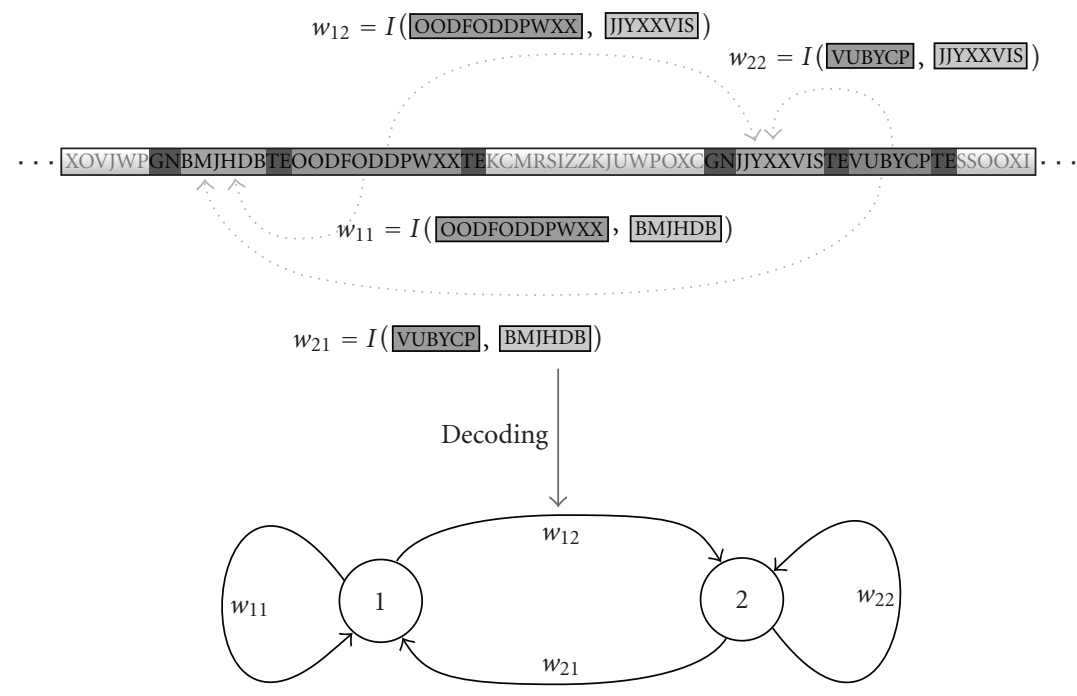

FIGURE 4: A simple artificial neural network represented with analog genetic encoding. The interaction strengths $w_{i j}$ are computed by the interaction map $I\left(s_{i}, s_{j}\right)$ which takes the sequence of characters $s_{i}$ associated to the output of neuron $i$ and the sequence of characters $s_{j}$ associated to the input of neuron $j$ as inputs.

recording sessions with six young healthy male subjects of a mean $( \pm$ SD $)$ age of $26( \pm 3)$ years. The subjects wore a Heally recording device (see Figure 2) for a total of 18 recording sessions which lasted 16 hours each and contained an overnight sleep. The datasets are composed of ECG and RSP recordings sampled at $100 \mathrm{~Hz}$ and $50 \mathrm{~Hz}$, respectively. The $a$ priori sleep and wake states of the subjects were determined by a trained technician who labeled the signals in 10-second intervals based on electromyogram, electrooculogram, and video recordings. The data were preprocessed and fed to the ANN. As in [7], the preprocessing step consisted of calculating the power spectrum of each signal using a shorttime fast Fourier transform with a window length of 40.96 seconds (see Figure 1(b)). For each of these segments, we calculated a feature vector as $\vec{v}=\log (\hat{S}(\omega))$, where $\hat{S}(\omega)$ is the periodogram of the segment. Experiments described in [8] revealed that frequency components above $10 \mathrm{~Hz}$ for ECG and $8 \mathrm{~Hz}$ for RSP do not contribute to the hand-designed classifier performance and can be removed. The resulting two input vectors are thus composed of 409 spectral inputs 


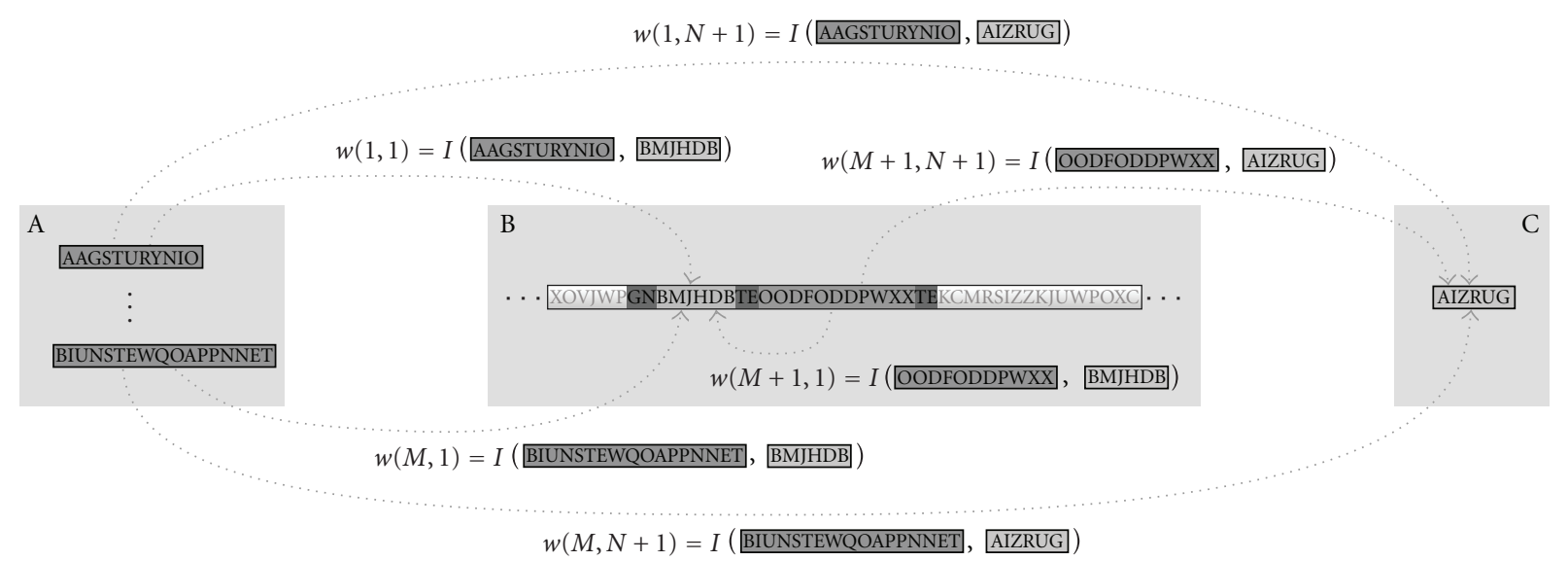

FIGURE 5: There are different ways to implement external inputs and outputs in AGE [28]. Here, the genome is split in three parts: (a) contains the coding sequences of the $M$ input neurons, (b) contains the definition of the $N$ hidden neurons, and (c) contains the regulatory sequence of the output neuron. In the decoding process, the coding sequences and the regulatory sequences of all neurons present in the genome are identified. The connection weights $w(x, y)$ can then be obtained by applying the interaction map to all pairs of coding sequences $x$ and regulatory sequences $y$.

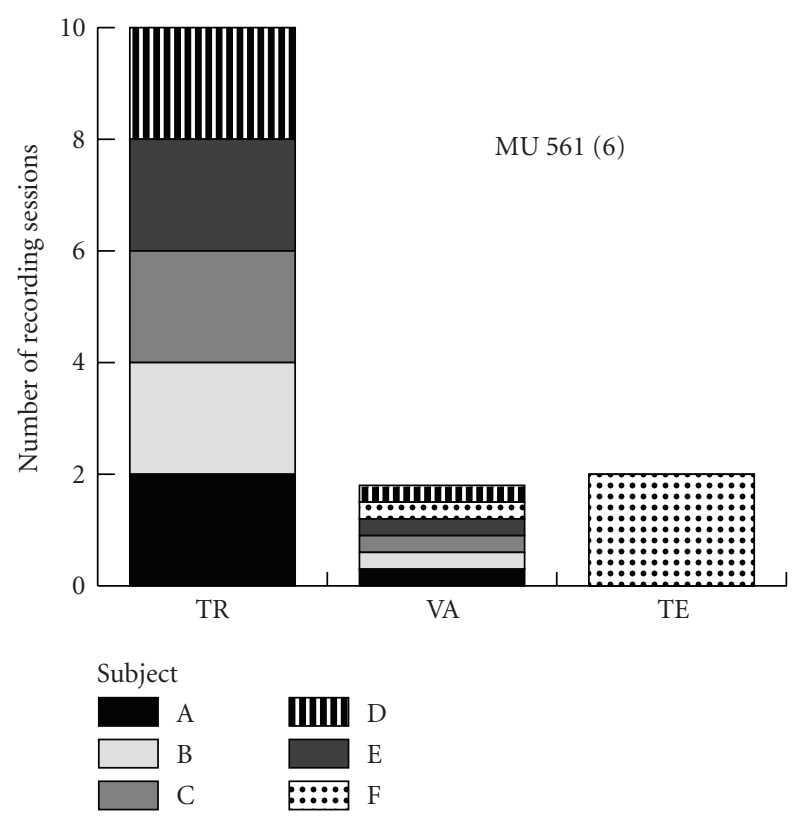

FIGURE 6: Distribution of the experimental data used for the training, validation, and test of the hand-designed and the evolutionary synthesized neural classifiers. The numbers indicate users in the training set (TR), users in the validation set (VA), and users in the test set (TE). There are six repetitions with different combinations of users/sessions in training, validation, and test sets.

from ECG and 327 spectral inputs from RSP. Together, they compose the set of 736 inputs that were fed to the ANN classifier (see Figure 1(c)).

3.2. Experimental Design. In order to evaluate the performance of the two classifiers, we divided the data into three different sets: a training set (TR), a validation set (VA), and a test set (TE) (see Figure 6). The training set contains a subset

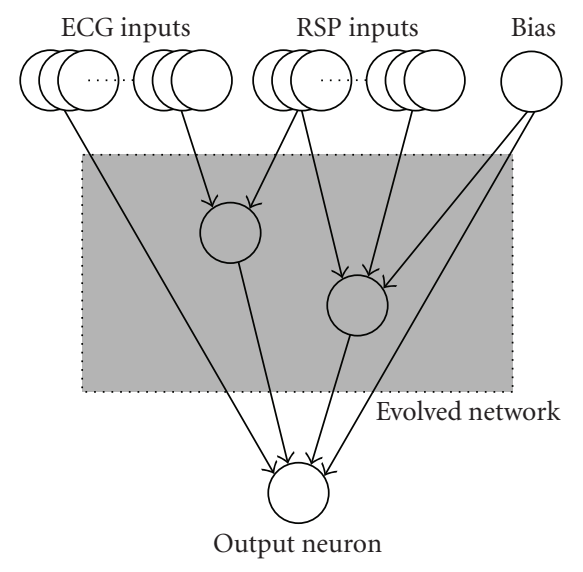

FIGURE 7: The neural classifier is automatically synthesized with analog genetic encoding. The evolved network can connect to an arbitrary subset of the 409 inputs from the ECG data, the 327 inputs from the RSP data and a bias unit. As the size of the network is not fixed, the number of hidden units in the network can increase or decrease over the course of evolution. The output unit indicates sleep or wake states using a simple threshold at an activation level of zero.

of the data from five of the six subjects. The validation set is composed of 2 hours of data from each subject, randomly sampled over the two available sessions and containing an equal amount of samples labeled as sleep and wake. This data is not used for training or for testing. The test set contains data from the subject that has not been used in the training. Five independent runs of each experiment are performed from different randomly assigned initial conditions. In order to prevent performance biases due to the choice of sessions, we repeat each experiment with all possible combinations of users in the test and trainingsets, making sure that the same sessions do not appear both in the training and in the 


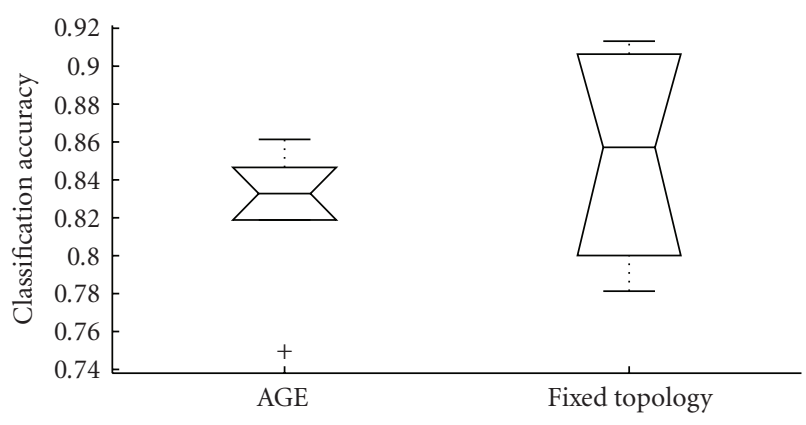

Figure 8: The average classification accuracy of the evolved networks (AGE) and the fixed topology networks trained with back propagation (fixed topology). The midline in each box is the median, the borders of the box represent the upper and the lower quartiles. The whiskers outside the box represent the minimum and maximum values obtained, except when there are outliers which are shown as small crosses. We define outliers as data points which differ more than 1.5 times the interquartile range from the border of the box. The notches permit the assessment of the significance of the differences of the medians. When the notches of two boxes overlap, the corresponding medians are not significantly different at (approximately) the 95\% confidence level [34].

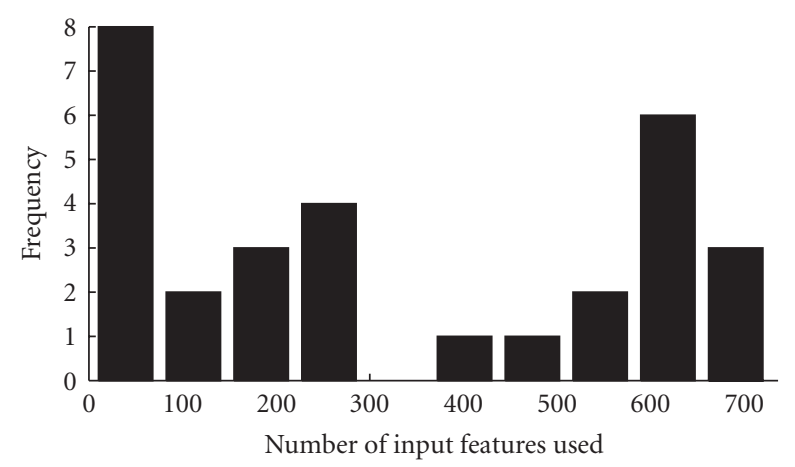

Figure 9: Histogram of the number of input features used by the evolved networks in the five repetitions of each of the six training cases. From the 30 networks, 8 used from 3 to 45 inputs, 2 used from 95 to 113 inputs, 3 used from 162 to 196 inputs, 4 used from 231 to 282 inputs, 1 used 411 and 1 used 484 inputs, 2 networks used from 522 to 533 inputs, 6 networks used from 602 to 647 inputs, and 3 networks used from 628 to 732 inputs.

testsets. This leads to a total of six different cases with five independent replications for each case.

\subsection{Algorithms}

3.3.1. Hand-Designed Fixed Topology Network. As a baseline for the classification accuracy, we used a feed-forward ANN with no hidden layers and a single output unit with a tangent-sigmoid transfer function. Additional experiments not reported here showed that the use of ANNs with a hidden layer does not improve the performance of the classifier. A similar finding has been reported by [30]. The synaptic weights of this fixed topology network were initialized with the Nguyen-Widrow method [31] and trained with a Levenberg-Marquardt back-propagation algorithm [32].

3.3.2. Network Synthesized with AGE. For the automatic synthesis of the network topology and parameters, the AGE representation was combined with a standard genetic algorithm (see [24] for more details). Using the abovementioned possibility of feature selection, the evolved network could connect to an arbitrary subset of the 409 inputs from the ECG data, the 327 inputs from the RSP data, and a constant bias unit (see Figure 7). Additionally, the evolutionary process might insert hidden neurons in the network in order to generate more complex network structures. The activation $y_{i}$ of the hidden neuron $i$ was computed as

$$
y_{i}=\sigma_{i}\left(\sum_{k=1}^{N} w(i, k) y_{k}+\sum_{l=1}^{M} w(i, N+l) I_{l}+w(i, N+M+1)\right),
$$

where $N$ is the number of hidden neurons in the network, $w(x, y)=w_{x y}$ are the entries of the weight matrix, $M=736$ is the number of available inputs, $I_{l}$ is the value of input $l$, and

$$
\sigma_{i}(z)=\frac{2}{1+e^{-\alpha_{i} z}}-1,
$$

is a sigmoid transfer function with slope parameter $\alpha_{i}$. The activation of the output neuron was computed analogously to the activation of the hidden neurons. The slope parameters $\alpha_{i}$ for the hidden neurons were encoded using the center of mass encoding [33].

Selection was performed using tournament selection and elitism. The algorithm parameters and mutation probabilities are listed in Table 1. In order to prevent bootstrap problems, the population was initialized with the best 100 networks out of 1000 randomly created genomes. Additionally, to save computation time, only a randomly selected subset of $10 \%$ of each training set was used for training. However, validation and testingwere always performed using $100 \%$ of the respective dataset. For each evolutionary run, the synthesized network was the network with the best performance on the validation set, in the collection of all the best performing networks observed at each of the 1000 generations that compose a run.

For both the back-propagation training and the evolutionary process, the measure of quality of the classifier was the sum over the data points of the squares of the difference between the actual and the desired classifier output.

\section{Results and Discussion}

As shown in Figure 8, the evolved networks and the fixed topology networks trained with back propagation do not display a significantly different classification accuracy (Wilcoxon rank sum test $P=.48$ ). However, while the hand-designed fixed topology networksemploy all of the 736 input features, many of the evolved networks used a 
TABLE 1: The parameters used in the evolutionary algorithm.

\begin{tabular}{lc}
\hline Parameter & Value \\
\hline Population size & 100 \\
Tournament size & 2 \\
Elite size & 1 \\
Recombination probability & .1 \\
Probability of character substitution (per character) & .001 \\
Probability of character insertion (per character) & .001 \\
Probability of character deletion (per character) & .0015 \\
Probability of fragment transposition & .01 \\
Probability of fragment duplication & .01 \\
Probability of fragment deletion & .015 \\
Probability of neuron insertion & .01 \\
\hline
\end{tabular}

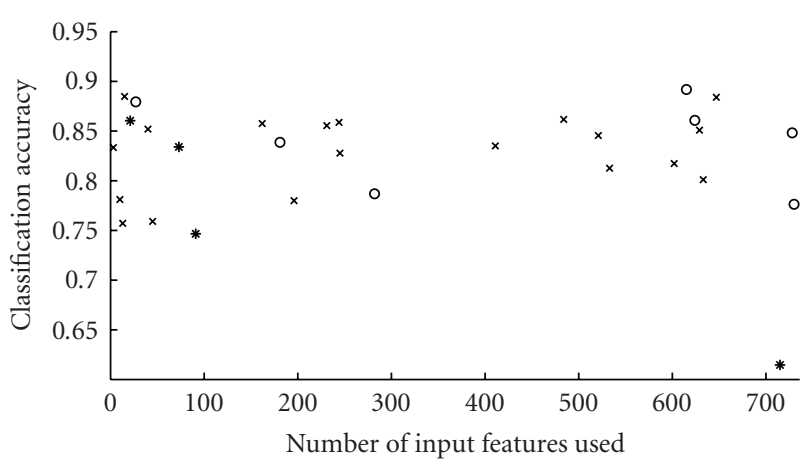

FIgURE 10: The performance of the evolved networks in the five repetitions of each of the six training cases. The horizontal axis represents the number of input features used by the network and the vertical axis gives the corresponding classification performance. The symbols indicate the number neurons in the hidden layer of the network. A cross indicates 0 hidden neurons, a circle indicates 1 hidden neuron, a star indicates 2 hidden neurons. Both the number of inputs and the number of hidden neurons are not significantly correlated with classification accuracy (see text).

drastically reduced set of inputs (see Figure 9, the median of the number of inputs used is 244.5). Figure 10 shows that there is no correlation between the number of inputs used by the evolved networks and their performance (Spearman's rank correlation coefficient $P=.02, P=.94)$. This indicates that many input features are indeed redundant and that it is possible to synthesize networks with a very small number of inputs which perform as well as the handdesigned network using all inputs. However, all networks use input features from both ECG and RSP data (see Figure 11). Given the results of [7], it is not surprising that the presence of both types of data is beneficial for the classification accuracy and thus selected during evolution. Note that in the evolutionary experiments, no additional penalty term was added to the objective function to bias the search toward small networks. This explains the presence of both networks using a significantly reduced set of inputs, and networks using almost the whole set of available inputs in the evolutionary results.

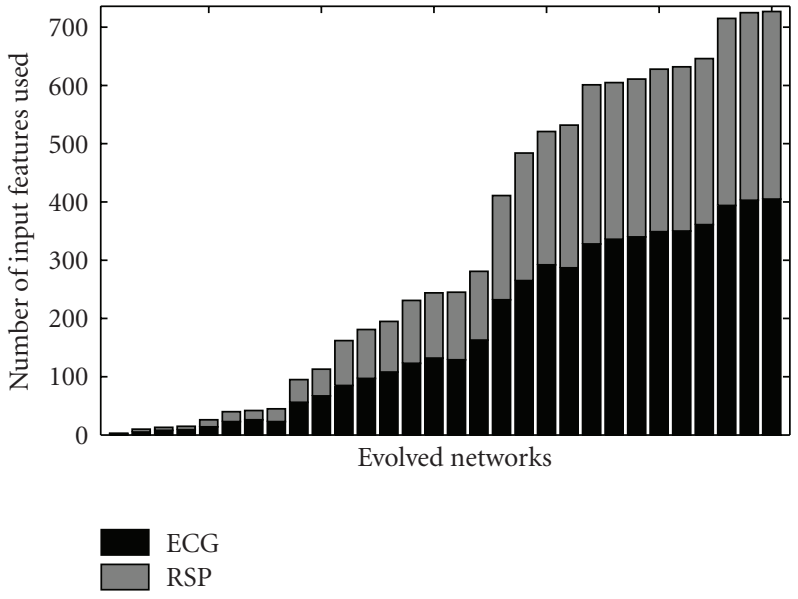

FIGURE 11: The evolved networks for the five repetitions of each of the six cases, sorted by the number of used input features. All networks use input features from both ECG and RSP data.

As mentioned above, the fixed topology network has no hidden layer. Of the 30 evolved networks, 19 feature no hidden neurons, 7 feature one hidden neuron, and 4 feature two hidden neurons. However, there is no correlation between the number of hidden neurons and the classification accuracy (Spearman's rank correlation coefficient $P=-.06$, $P=.74)$. This substantiates the conjecture formulated in [7] that a hidden layer is not necessary for optimal performance in this task. Note, however, that this conjecture applies to this specific problem and does not extend to general classification applications.

\section{Conclusion}

Portable devices for biomedical signal analysis, like sleep/wake classification, have the potential to alleviate health problems and prevent accidents. Recent advances in sensor development and miniaturization allow for the construction of small mobile devices which integrate biomedical sensors and a microprocessor with sufficient processing power for many applications. However, one of the critical challenges, that remains, is the design of efficient classifiers which can be implemented on these small mobile systems. While the classification accuracy has to be as high as possible, the computational effort and thus the energy requirements for classification have to remain low. The results presented in this paper demonstrate that analog genetic encoding (AGE) permits the automatic evolutionary synthesis of compact neural classifiers for the problem of sleep/wake classification. Compared to a hand-designed classifier trained with back propagation, the possibility of the evolutionary selection of a subset of the available inputs permits a drastic reduction of the number of inputs without significant degradation of the classifier performance. For example, in the experiments presented here, the evolutionary synthesis with AGE found a classifier with the accuracy of $88.49 \%$, using only 15 of the 736 input features used by the hand-designed network. The implementation of this 
evolved solution on a digital signal controller of the dsPIC33 product family (Microchip Technology Inc., USA) requires only $5.13 \%$ of the instructions used by an implementation of the hand-designed network on the same processor. This is a reduction of the computational cost of almost $95 \%$. Moreover, the savings in computational cost and energy can be increased even further by adapting the sensory modalities and preprocessing steps to the reduced set of input features.

\section{Acknowledgments}

This work was supported by the Swiss National Science Foundation, Grant no. 200021-112060 and the Solar Impulse Project grant of Ecole Polytechnique Fédérale de Lausanne (EPFL). Thanks to Daniel Marbach and Sara Mitri for their comments on an earlier version of this manuscript and the anonymous reviewers for their helpful suggestions.

\section{References}

[1] D. E. Rumelhart, G. E. Hinton, and R. J. Williams, "Learning representations by back-propagation of errors," Nature, vol. 323, pp. 533-536, 1986.

[2] D. Floreano, P. Dürr, and C. Mattiussi, "Neuroevolution: from architectures to learning," Evolutionary Intelligence, vol. 1, no. 1, pp. 47-62, 2008.

[3] C. Mattiussi and D. Floreano, "Analog genetic encoding for the evolution of circuits and networks," IEEE Transactions on Evolutionary Computation, vol. 11, no. 5, pp. 596-607, 2007.

[4] A. Rechtschaffen, A. Kales, R. Berger, and W. Dement, A Manual of Standardized Terminology, Techniques and Scoring System for Sleep Stages of Human Subjects, Public Health Service, U.S. Government Printing Office, Washington, DC, USA, 1968.

[5] A. Sadeh and C. Acebo, "The role of actigraphy in sleep medicine," Sleep Medicine Reviews, vol. 6, no. 2, pp. 113-124, 2002.

[6] C. P. Pollak, W. W. Tryon, H. Nagaraja, and R. Dzwonczyk, "How accurately does wrist actigraphy identify the states of sleep and wakefulness?" Sleep, vol. 24, no. 8, pp. 957-965, 2001.

[7] W. Karlen, C. Mattiussi, and D. Floreano, "Adaptive sleep/wake classification based on cardiorespiratory signals for wearable devices," in Proceedings of the IEEE on Biomedical Circuits and Systems Conference (BIOCAS '07), pp. 203-206, Montreal, Canada, November 2007.

[8] W. Karlen, C. Mattiussi, and D. Floreano, "Sleep and wake classification with ECG and respiratory effort signals," to appear in IEEE Transactions on Biomedical Circuits and Systems.

[9] R. D. Ogilvie, "The process of falling asleep," Sleep Medicine Reviews, vol. 5, no. 3, pp. 247-270, 2001.

[10] G. P. Zhang, "Neural networks for classification: a survey," IEEE Transactions on Systems, Man and Cybernetics, Part C, vol. 30, no. 4, pp. 451-462, 2000.

[11] M. Rocha, P. Cortez, and J. Neves, "Evolution of neural networks for classification and regression," Neurocomputing, vol. 70, no. 16-18, pp. 2809-2816, 2007.

[12] M. Čepek, M. Šnorek, and V. Chudáček, "ECG signal classification using GAME neural network and its comparison to other classifiers," in Proceedings of the 18th International Conference on Artificial Neural Networks (ICANN '08), vol.
5163 of Lecture Notes in Computer Science, pp. 768-777, Prague, Czech Republic, September 2008.

[13] L. Chen and D. Alahakoon, "NeuroEvolution of augmenting topologies with learning for data classification," in Proceedings of the International Conference on Information and Automation (ICIA '06), pp. 367-371, Shandong, China, December 2006.

[14] X. Yao, "Evolving artificial neural networks," Proceedings of the IEEE, vol. 87, no. 9, pp. 1423-1447, 1999.

[15] K. O. Stanley and R. Miikkulainen, "Evolving neural networks through augmenting topologies," Evolutionary Computation, vol. 10, no. 2, pp. 99-127, 2002.

[16] R. S. Zebulum, M. Vellasco, and M. A. Pacheco, "Variable length representation in evolutionary electronics," Evolutionary Computation, vol. 8, no. 1, pp. 93-120, 2000.

[17] F. Gruau, "Automatic definition of modular neural networks," Adaptive Behavior, vol. 3, no. 2, pp. 151-183, 1994.

[18] J. R. Koza, Genetic Programming II: Automatic Discovery of Reusable Programs, MIT Press, Cambridge, Mass, USA, 1994.

[19] J. Bongard, "Evolving modular genetic regulatory networks," in Proceedings of the Congress on Evolutionary Computation (CEC'02), vol. 2, pp. 1872-1877, Honolulu, Hawaii, USA, May 2002.

[20] T. Reil, "Dynamics of gene expression in an artificial genomeimplications for biological and artificial ontogeny," in Proceedings of the 5th European Conference on Artificial Life (ECAL '99), pp. 457-466, Lausanne, Switzerland, September 1999.

[21] T. Reil, "Artificial genomes as models of gene regulation," in On Growth, Form and Computers, pp. 256-277, Academic Press, London, UK, 2003.

[22] C. Mattiussi, D. Marbach, P. Dürr, and D. Floreano, "The age of analog networks," AI Magazine, vol. 29, no. 3, pp. 63-76, 2008.

[23] J. Reisinger and R. Miikkulainen, "Acquiring evolvability through adaptive representations," in Proceedings of the 9th Annual Genetic and Evolutionary Computation Conference (GECCO '07), pp. 1045-1052, ACM Press, London, UK, July 2007.

[24] P. Dürr, C. Mattiussi, and D. Floreano, "Neuroevolution with analog genetic encoding," in Proceedings of the 9th International Conference on Parallel Problem Solving from Nature (PPSN '06), vol. 9, pp. 671-680, Springer, Reykjavik, Iceland, September 2006.

[25] A. Soltoggio, P. Dürr, C. Mattiussi, and D. Floreano, "Evolving neuromodulatory topologies for reinforcement learning-like problems," in Proceedings of the IEEE Congress on Evolutionary Computation (CEC '07), P. Angeline, M. Michaelewicz, G. Schonauer, X. Yao, and Z. Zalzala, Eds., pp. 2471-2478, IEEE Press, Singapore, September 2007.

[26] P. Dürr, C. Mattiussi, A. Soltoggio, and D. Floreano, "Evolvability of neuromodulated learning for robots," in Proceedings of the ECSIS Symposium on Learning and Adaptive Behaviors for Robotic Systems (LAB-RS '08), pp. 41-46, Edinburgh, Scotland, August 2008.

[27] G. Gusfield, Algorithms on Strings, Trees, and Sequences, Cambridge University Press, Cambridge, UK, 1997.

[28] C. Mattiussi, Evolutionary synthesis of analog networks, Ph.D. dissertation, EPFL, Lausanne, Switzerland, 2005.

[29] A. Wagner, "Robustness, evolvability, and neutrality," FEBS Letters, vol. 579, no. 8, pp. 1772-1778, 2005.

[30] J. Principe and A. Tome, "Performance and training strategies in feedforward neural networks: an application to sleep scoring," in Proceedings of the International Joint Conference on Neural Networks (IJCNN '89), vol. 1, pp. 341-346, Washington, DC, USA, June 1989. 
[31] D. Nguyen and B. Widrow, "Improving the learning speed of 2-layer neural networks by choosing initial values of the adaptive weights," in Proceedings of International Joint Conference on Neural Networks (IJCNN '90), pp. 21-26, San Diego, Calif, USA, June 1990.

[32] M. T. Hagan and M. B. Menhaj, "Training feedforward networks with the Marquardt algorithm," IEEE Transactions on Neural Networks, vol. 5, no. 6, pp. 989-993, 1994.

[33] C. Mattiussi, P. Dürr, and D. Floreano, "Center of mass encoding: a self-adaptive representation with adjustable redundancy for real-valued parameters," in Proceedings of the 9th Annual Genetic and Evolutionary Computation Conference (GECCO '07), pp. 1304-1311, London, UK, July 2007.

[34] R. McGill, J. W. Tukey, and W. A. Larsen, "Variations of box plots," The American Statistician, vol. 32, no. 1, pp. 12-16, 1978. 